\title{
Application d'une électrode sensible à la pression partielle du dioxyde de carbone au dosage " in situ " de ce composant dans quelques produits laitiers fermentés
}

\author{
J.O. BOSSET, J.-P. PAUCHARD et R. GAUCH \\ Station fédérale de recherches laitières, $\mathrm{CH}$-3097 Liebefeld-Berne, Suisse
}

\begin{abstract}
Résumé
Le présent travail a pour objet la mise au point d'une méthode de mesurage de la pression partielle du gaz carbonique $\left(\mathrm{pCO}_{2}\right)$ dans certains produits laitiers fermentés tels que yoghourts, kéfirs et boissons acidifiées. Il évalue les possibilités et les limites d'application de cette nouvelle méthode et la compare à une méthode de référence, soit un dosage photométrique non dispersif dans l'infrarouge. La simplicité, la rapidité (environ 10 déterminations/heure), la répétabilité (1 à $3 \%$ relatifs) ainsi que l'exactitude (pas d'interférence pour les produits laitiers considérés) de la méthode proposée, de même que le coût relativement modique de l'équipement requis la destinent à des dosages de routine. Au nombre des applications possibles de cette méthode, on peut citer tant les contrôles de fabrication et de stockage de ces produits laitiers que des mesures spéciales, telles celles de la perméabilité au $\mathrm{CO}_{2}$ de leur matériau d'emballage.

Mots clés : Dosage du gaz carbonique - Electrode sensible à la $\mathrm{pCO}_{2}$ - Produits laitiers fermentés.
\end{abstract}

\section{Summary}

\section{Determination of carbon dioxide in various fermented milk products using} a gaz sensing $\mathrm{CO}_{2}$ - electrode

A method has been developed for measuring the partial pressure of carbon dioxide $\mathrm{pCO}_{2}$ in various fermented milk products such as yoghurts, kefirs and acid drinks. The possibilities and limits of this new method have been evaluated. The method was compared to a reference method i.e. non dispersive photometric measurement in the infra-red. The method is simple, rapid (approx. 10 determinations/h) and accurate (no interference from lactic acid, volatile fatty acids, etc.). The repeatability is 1-3\%. The relatively low cost of equipment required renders this method suitable for routine measurements in milk products $\left(\mathrm{CO}_{2}\right.$ content or production in kefir, yoghurt, etc.) during manufacture and storage. The method is also applicable to other determinations such as the measurement of the permeability of various packaging materials.

Key words : Determination of carbone dioxide - Gas sensing $\mathrm{CO}_{2}$-electrod - Fermented milk products. 


\section{Introduction}

La teneur en gaz carbonique des produits laitiers fermentés peut donner de précieuses indications technologiques pour le contrôle tant de leur fabrication (par exemple kéfirs) que de leur entreposage (kéfirs, yoghourts). Pendant ce dernier, un accroissement de la concentration en $\mathrm{CO}_{2}$ est en effet signe d'altération (activité de levures, éventuellement de moisissures) ; une diminution est en revanche une indication de perméabilité aux constituants gazeux $\left(\mathrm{CO}_{2}, \mathrm{O}_{2}\right)$ ou volatils (arômes).

Un grand nombre de méthodes ont déjà été proposées pour le dosage du gaz carbonique (cf. articles de revue : CHARLOT, 1966; KozLOWSKI et NAMIESNIK, 1979). Pour mémoire, on peut en rappeler les différents principes : titrages acidobasiques (ROBERTSON, 1962 ; FLUECKIGER et al., 1978), parfois après entraînement par un gaz inerte, mesurages enzymatiques (AOAC, 1980c ; CAPUT, 1971), volumétriques (AOAC, 1980b ; LIPKA et SCHOPFER, 1972), manométriques (AOAC, 1980a ; BRUNNer et al., 1977), gravimétriques (KNOTt et BELCHER, 1975), colorimétriques (Moll et al., 1975), chromatographiques (THompson, 1977 ; Simmonds, 1978) et photométriques non dispersifs dans l'infrarouge (IR) (WiedeKING et al., 1980 ; Bosset et al, 1980b; BLANC et al., 1980 ; PAUCHARD et al., 1980). Quelques travaux ont également proposé le recours à des méthodes électrochimiques potentiométriques, basées sur l'emploi d'électrodes dites sensibles à la pression partielle de ce gaz $\left(\mathrm{pCO}_{2}\right)$ (LONVAUD-FuneL et RiBEREAU-GAYON, 1976 ; LADENSON et al., 1975 ; Bosset et al., 1980a). Une étude récente fait même mention d'une application au lait (VAN DEN BERG, 1979). Bien que d'un emploi aisé, rapide, économique et sans danger, cette dernière méthode ne semble guère avoir été appliquée au dosage du $\mathrm{CO}_{2}$ dans des produits laitiers fermentés tels que yoghourts, « yoghourts pasteurisés », kéfirs et boissons lactées acidifiées. Le $\mathrm{pH}$ naturel de tels produits, généralement inférieur à 4,5 et bien tamponné, est pourtant idéal pour une utilisation directe de l'électrode à $\mathrm{pCO}_{2}$ (fig. 1).

La présente étude a pour objet la mise au point d'une méthode de mesure potentiométrique directe de la $\mathrm{pCO}_{2}$ de certains produits laitiers fermentés, l'évaluation des performances analytiques et la comparaison de cette méthode à une méthode de référence (dosage photométrique non dispersif dans l'IR ; BOSSET et al., 1980b) ainsi qu'une application à la détermination de la perméabilité au $\mathrm{CO}_{2}$ de quelques emballages utilisés pour de tels produits laitiers.

\section{Principe de la détermination potentiométrique de la $\mathrm{pCO}_{3}$}

Le principe de cette détermination (anonymes, $1982 \mathrm{a}$ et b ; SHODA et IsHIKAWA, 1981) consiste à remplacer le mesurage de la $\mathrm{pCO}_{2}$ de l'échantillon à analyser, à matrice généralement complexe, par le mesurage du $\mathrm{pH}$ d'une solution de bicarbonate située à l'intérieur de l'électrode à $\mathrm{pCO}_{2}$. Les $\mathrm{pCO}_{2}$ de ces deux milieux doivent préalablement s'être équilibrées par la diffusion de $\mathrm{CO}_{2}$ gazeux à travers la membrane semi-perméable de l'électrode. Aucun autre composant gazeux ou volatil acide susceptible d'interférer ne doit pouvoir diffuser. Un calibrage adéquat permet alors de convertir les valeurs de $\mathrm{pH}$ mesurées en $\mathrm{pCO}_{2}$. 


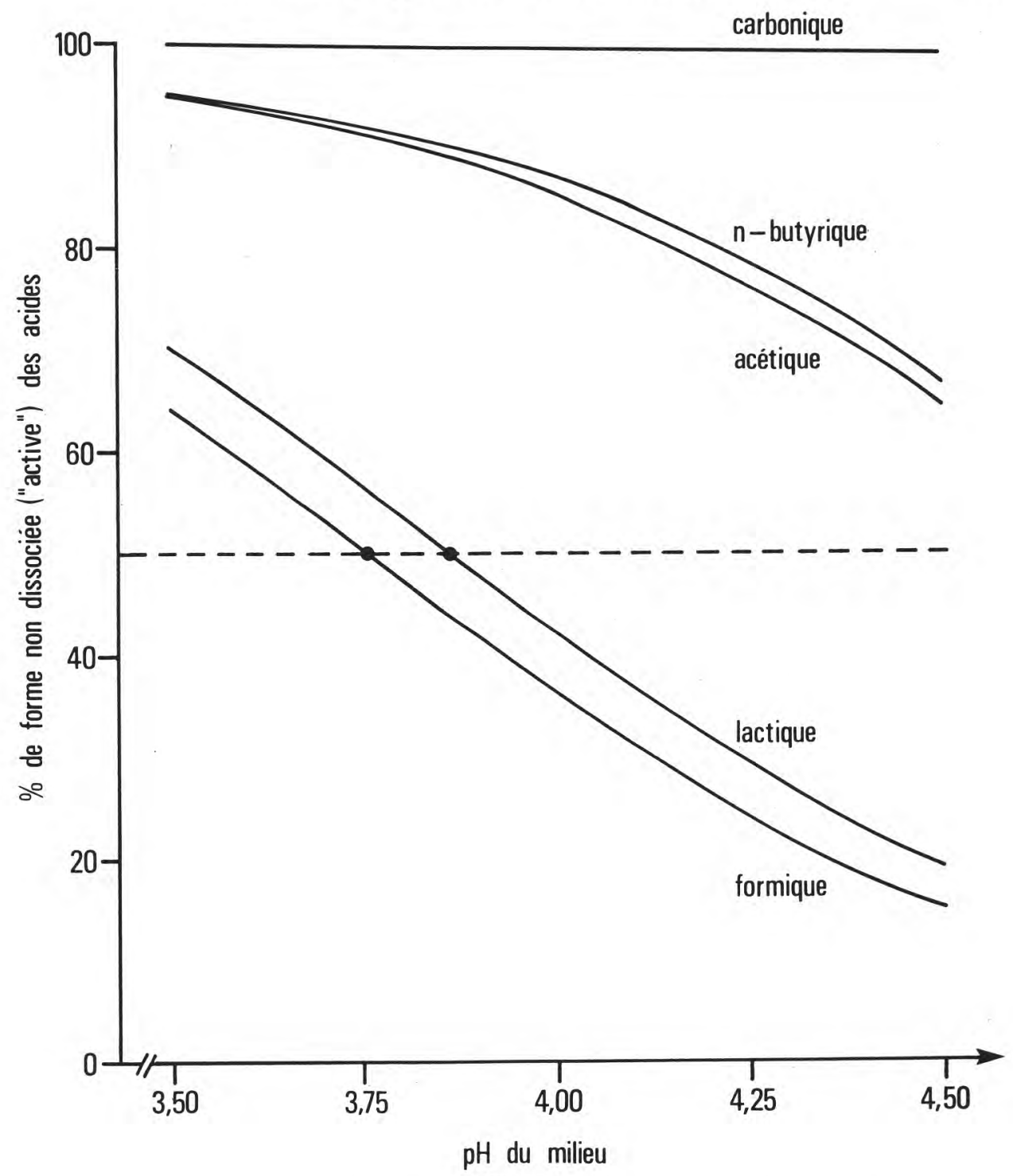

Fig. 1

Rapport (en \%) des formes non dissociée/dissociée de l'acide carbonique et de quelques acides carboxyliques susceptibles d'interférer avec le mesurage de la $\mathrm{pCO}_{2}$ en fonction $d u p H$.

Le domaine de $p H$ correspond à celui des produits laitiers fermentés considérés. Les valeurs de $p K$ suivantes ont été utilisées : 6,37 et 10,25 à $25^{\circ} C$ (ac. carbonique) ; 4,81 à $20^{\circ} \mathrm{C}$ (ac. butyrique) ; 4,75 à $25^{\circ} \mathrm{C}$ (ac. acétique) ; 3,86 à $25^{\circ} \mathrm{C}$ (ac. lactique) et 3,75 à $20^{\circ} \mathrm{C}$ (ac. formique).

pH value versus ratio (in \%) of undissociated to dissociated carbonic acid and some carboxylic acids which could interfere with $\mathrm{pCO}_{2}$ measurement.

The $p H$ range corresponds to that of the fermented milk products considered. The following $p K$ values were used : 6.37 and 10.25 at $25^{\circ} C$ for carbonic acid : 4.81 at $20^{\circ} C$ for n-butyric acid; 4.75 at $25^{\circ} \mathrm{C}$ for acetic acid; 3.86 at $25^{\circ} \mathrm{C}$ for lactic acid; 3.75 at $20^{\circ} \mathrm{C}$ for formic acid. 


\section{Dispositif expérimental}

A. Appareillage de mesure de la $\mathrm{pCO}_{2}$ des échantillons

L'appareillage utilisé se compose d'une électrode potentiométrique dite «spécifique " ou "sensible » à la $\mathrm{pCO}_{2}$, de type industriel, à manchon d'acier de $150 \mathrm{~mm}$ de long (réf. 780/35/3M/-1530 de INGOLD AG, CH-8902 Urdorf) et d'un amplificateur de mesure 0-300 mbar à affichage digital avec trois chiffres significatifs (type INGOLD IL 525 du même fournisseur). La sortie analogique de l'amplificateur est pontée par une résistance de $50 \Omega$ pour permettre un raccordement à un enregistreur linéaire LINSEIS 0-1V (GLONNER-ELECTRONIC AG, CH-5305 Wuerenlingen). Sans être indispensable au mesurage, cet enregistreur en continu facilite néanmoins la lecture du résultat en indiquant à quel moment l'équilibre de diffusion est atteint (plateau, cf. fig. 2). Le brassage de l'échantillon, indispensable pour accélérer la mise en équilibre des $\mathrm{pCO}_{2}$ mais insuffisant pour briser le gel des produits dits «fermes », est assuré par un agitateur adapté à la viscosité des milieux mesurés et par un barreau magnétique adéquat (par ex. réf. 2'0276 de SEMADENI AG, $\mathrm{CH}-3072$ Ostermundigen : longueur : $35 \mathrm{~mm}$, diamètre : $9 \mathrm{~mm}$, à anneau médian). La figure 2 indique

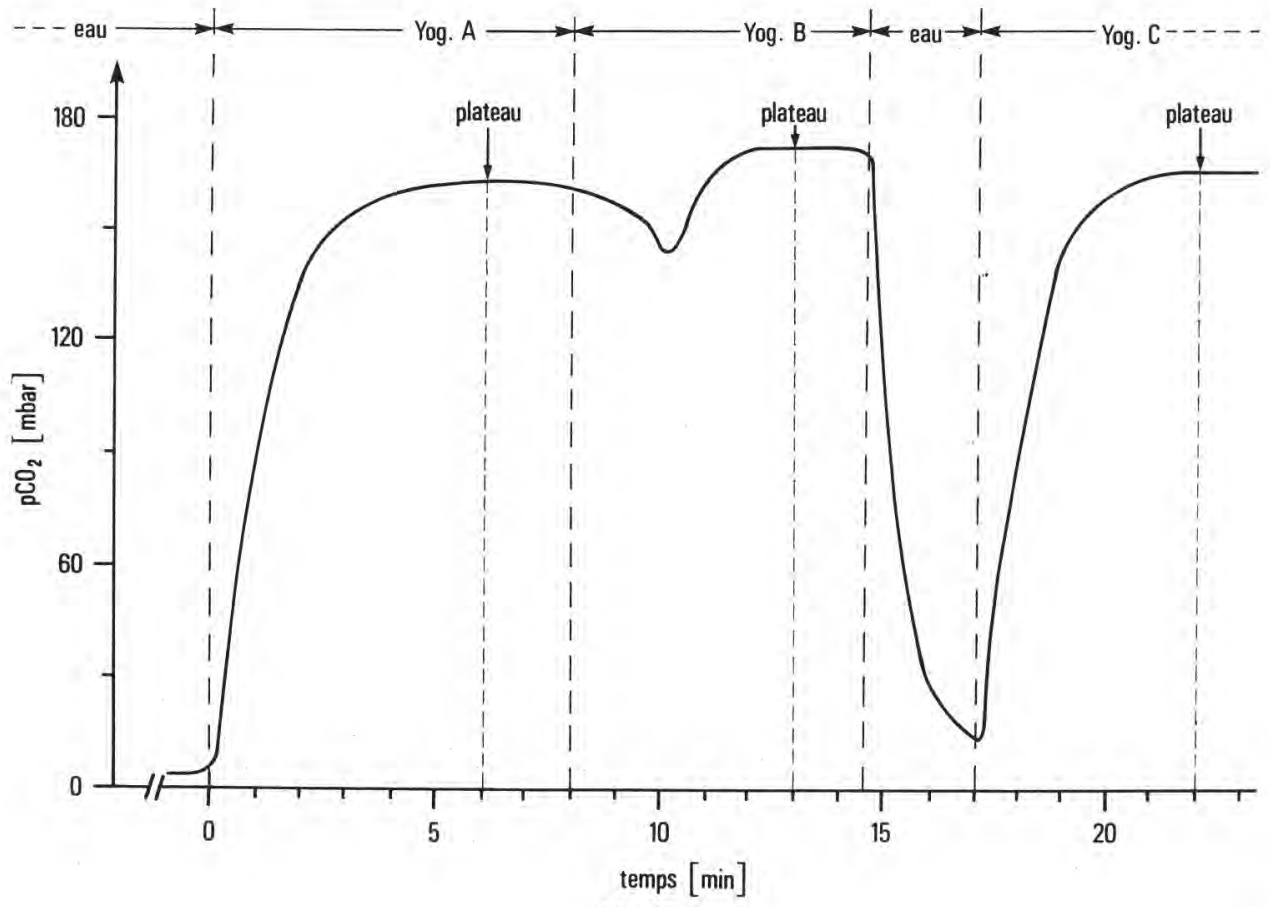

Fig. 2

Réponse typique d'une électrode lors de la mesure de la $\mathrm{pCO}_{2}$ de trois échantillons différents de yoghourt $(A, B, C)$ sans et avec rinçage de l'électrode à l'eau distillée.

Typical electrode response during the measurement of $\mathrm{pCO}_{2}$ of three different yoghurt samples $(A, B, C)$ with and without rinsing of the electrode with distilled water. 
l'allure générale des graphes obtenus avec et sans rinçage à l'eau distillée de l'électrode à $\mathrm{pCO}_{2}$.

\section{B. Appareillage de mesure du $p H$ des échantillons}

La mesure simultanée des valeurs de $\mathrm{pH}$ des échantillons est assurée par un pH-mètre type 605 de METROHM AG, CH-9100 Herisau, équipé d'une électrode combinée de verre.

\section{Choix des échantillons}

Pour mettre au point la méthode, un certain nombre de yoghourts nature $(1 \times 10$, puis $1 \times 20)$, provenant chaque fois d'un même lot homogène, ont été prélevés pour étudier l'influence du mode opératoire utilisé (avec ou sans purge avec $\mathrm{N}_{2}$ de l'espace de tête) ainsỉ que la répétabilité des mesures.

Pour tester la méthode et la comparer à la méthode de référence, 51 échantillons représentant 17 produits laitiers fermentés frais (cf. tabl. 1), tels que yoghourts nature et aux fruits, entiers et partiellement écrémés, fermes ou brassés, kéfirs et boissons acidifiées, ont été prélevés chaque fois en triple (même date de fabrication) dans une grande entreprise de fabrication et de distribution.

Tous les échantillons susmentionnés, d'un poids au remplissage de $180 \mathrm{~g}$, avaient pour emballage un gobelet de polystyrène transparent incolore (cf. description détaillée ci-après sous lettre e).

Environ 60 yoghourts nature provenant d'une seule et même charge, de $180 \mathrm{~g}$ également, ont encore été utilisés pour déterminer la perméabilité au $\mathrm{CO}_{2}$ des cinq types d'emballage suivants :

a) Un pot de verre $(119 \mathrm{~g})$ transparent, fortement teinté brun, avec un couvercle souple réutilisable en polyéthylène blanc $(2,8 \mathrm{~g})$; abrégé $\mathrm{V} / \mathrm{B}$;

b) Un pot de verre $(119 \mathrm{~g})$ transparent incolore avec un couvercle d'aluminium scellé à chaud $(0,8 \mathrm{~g})$ et présentant latéralement une petite étiquette de papier ; abrégé : V/I ;

c) Un gobelet $(7 \mathrm{~g})$ en polystyrène transparent, fortement teinté brun dans la masse, avec un couvercle d'aluminium scellé à chaud $(0,6 \mathrm{~g})$; abrégé : PS/B ;

d) Un gobelet $(8 \mathrm{~g})$ en polystyrène cartonné (emballage du type « $\mathrm{K} 3$ », «double couche $)$, pratiquement opaque avec le même couvercle d'aluminium scellé à chaud que PS/B $(0,6 \mathrm{~g})$; abrégé : PS/C ;

e) Un gobelet $(7 \mathrm{~g})$ en polystyrène transparent incolore, avec le même couvercle d'aluminium scellé à chaud que PS/B $(0,6 \mathrm{~g})$; abrégé : PS/I.

\section{Mode opératoire détaillé}

L'électrode à $\mathrm{pCO}_{2}$ et l'amplificateur de mesure correspondant sont calibrés préalablement (en mbar) selon les instructions du manuel (anonyme, 1982a) à l'aide de deux solutions tampons $\mathrm{pH}$ (sol. $\mathrm{A}$ : réf. $33780-1038 ; \mathrm{pH}$ mesuré $=$ 6,$665 ; \mathrm{pCO}_{2}=63$ mbar à $22^{\circ} \mathrm{C}$ et sol. $\mathrm{B}$. : réf. $33780-1039 ; \mathrm{pH}$ mesuré $=$ 7,$880 ; \mathrm{pCO}_{2}=3,6$ mbar à $22^{\circ} \mathrm{C}$ ) livrées par le fabricant. Les échantillons sont 
Valeurs de $\mathrm{pH}$ et de $\mathrm{pCO}_{2}$ et teneurs en $\mathrm{CO}_{2}$ total de quelques produits laitiers fermentés du commerce $p \mathrm{H}, \mathrm{pCO}_{2}$ values and total $\mathrm{CO}_{2}$ content of some commercial fermented milk products

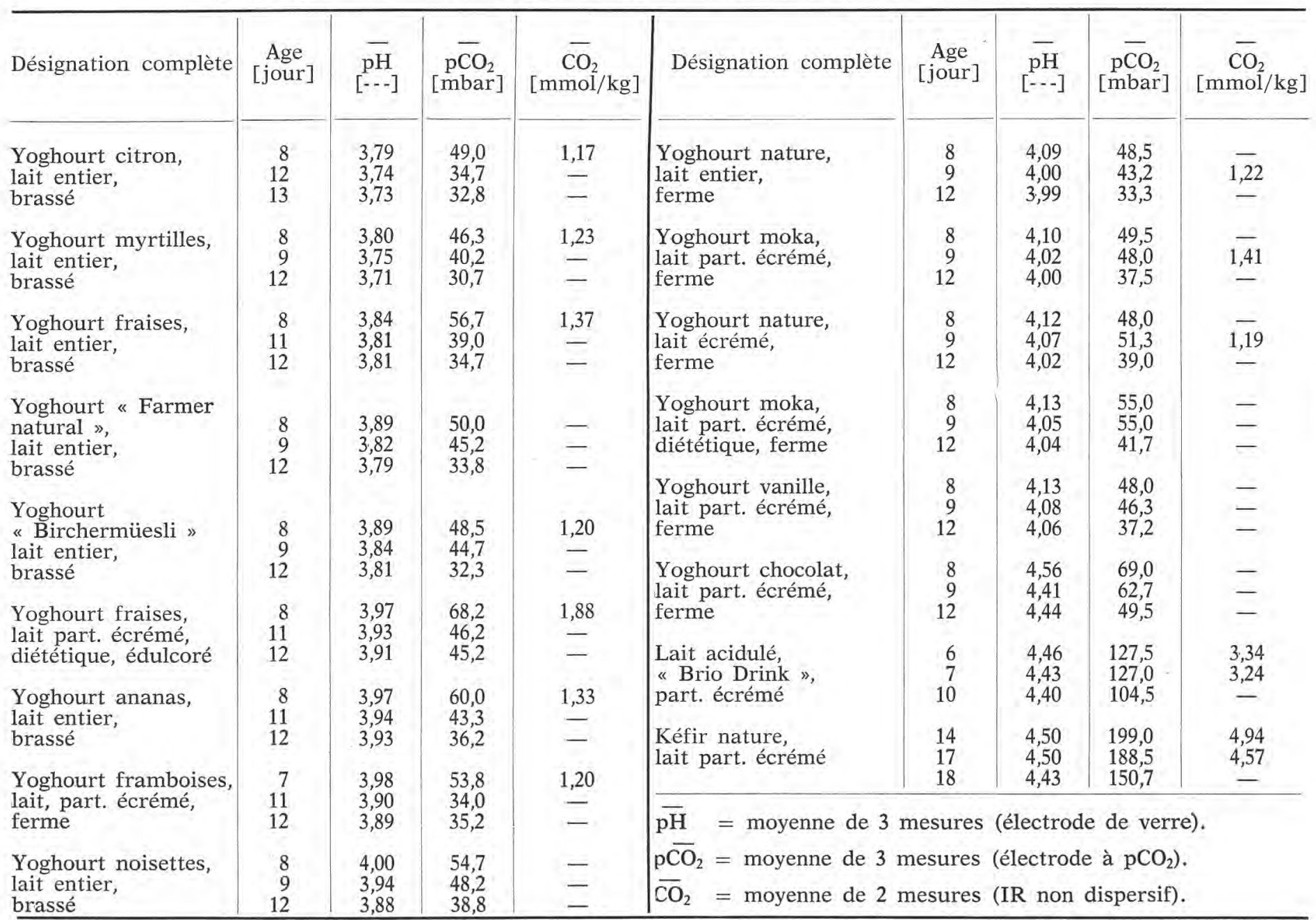


thermostatés à $22,0^{\circ} \mathrm{C} \pm 0,5^{\circ} \mathrm{C}$ pendant une heure au moins. La température joue en effet un rôle important, notamment sur la solubilité du $\mathrm{CO}_{2}$ dans l'échantillon et sur la pente de l'électrode interne à $\mathrm{pH}$ (équation de Nernst).

Pour la préparation des échantillons, le présent travail propose deux modes opératoires, soit avec (nécessaire en cas de détermination simultanée de la $\mathrm{pO}_{2}$ ) ou sans purge à l'azote de l'espace de tête de ces derniers. Si l'on choisit la variante avec purge de l'espace de tête, on perce dans le couvercle deux petits trous $(\varnothing=1-2 \mathrm{~mm})$ diamétralement opposés, par lesquels on fait passer un flux d'azote de 1 à $2 \mathrm{ml} / \mathrm{s}$ pendant environ $60 \mathrm{~s}$. On les obture immédiatement après au moyen d'un morceau de ruban adhésif.

Pour le mesurage à proprement parler, on secoue vigoureusement pendant $60 \mathrm{~s}$ le gobelet encore fermé et thermostaté, purgé ou non, afin de briser le gel des yoghourts fermes et rendre homogène le contenu en $\mathrm{CO}_{2}$ de la masse de l'échantillon (ce point est surtout important pour les échantillons obtenus dans des emballages perméables au $\mathrm{CO}_{2}$, qui peuvent présenter d'importants gradients de $\mathrm{pCO}_{2}$ ). On enlève alors le couvercle. On introduit successivement le barreau magnétique et l'électrode à $\mathrm{pCO}_{2}$. On fixe verticalement cette dernière à un support au moyen d'une pince, de façon à ce que sa membrane se situe immergée à environ $1,5 \mathrm{~cm}$ au-dessus du barreau magnétique : une distance inférieure accroît le risque d'endommager la membrane de l'électrode de verre et la membrane semi-perméable de l'électrode à $\mathrm{pCO}_{2}$; une distance supérieure accroît celui de perdre du $\mathrm{CO}_{2}$ et nuit à la mise en équilibre des $\mathrm{pCO}_{2}$. Un repère horizontal préalablement tracé sur le manchon de l'électrode garantit à cette fin une immersion répétable et sans danger. Le brassage de l'échantillon est réglé de manière à ce que la surface visible de ce dernier apparaisse légèrement ridée et déformée, sans qu'il ne se forme toutefois un cône d'aspiration. (Il importe que le barreau magnétique ne circule pas orbitalement au fond du gobelet, mais pivote bien sur son axe, ce que l'on peut déterminer grâce au «bruit » produit par le barreau magnétique). On attend alors que la valeur de la $\mathrm{pCO}_{2}$ affichée se stabilise (par ex. plateau sur l'enregistreur, cf. fig. 2) pour la noter.

La dérive journalière de l'électrode est insignifiante, tant que la membrane reste mécaniquement intacte et chimiquement inaltérée.

\section{Résultats expérimentaux et discussion}

A. Influence du mode opératoire sur la détermination et étude de la répétabilité (1) et de la reproductibilité (1) des mesures

L'influence de la purge à l'azote décrite ci-avant, indispensable en cas de détermination simultanée des pressions partielles du gaz carbonique $\left(\mathrm{pCO}_{2}\right)$ et de l'oxygène $\left(\mathrm{pO}_{2}\right)$ dans le même échantillon, entraîne - dans les conditions définies ci-avant - un abaissement relatif de la $\mathrm{pCO}_{2}$ de l'ordre de 13 à $16 \%$ (cf. tabl. 2 et 3 ). Cet abaissement est certainement dû au fait que le $\mathrm{CO}_{2}$ de l'espace de tête qui est entraîné dans la purge (analytiquement parlant cette fraction n'appartient d'ailleurs pas à l'échantillon) est remplacé, probablement

(1) Les termes de répétabilité " et de la « reproductibilité » ne sont pas utilisés ici dans leur acception usuelle. 


\section{TABLEAU II}

Influence de la purge à l'azote de l'espace de tête des yoghourts sur les mesures de la $\mathrm{pCO}_{2}$

The influence of flushing of the headspace with $\mathrm{N}_{2}$ on $\mathrm{pCO}_{2}$ measurements (10 one week old yoghurts from a homogeneous lot were used; single determination)

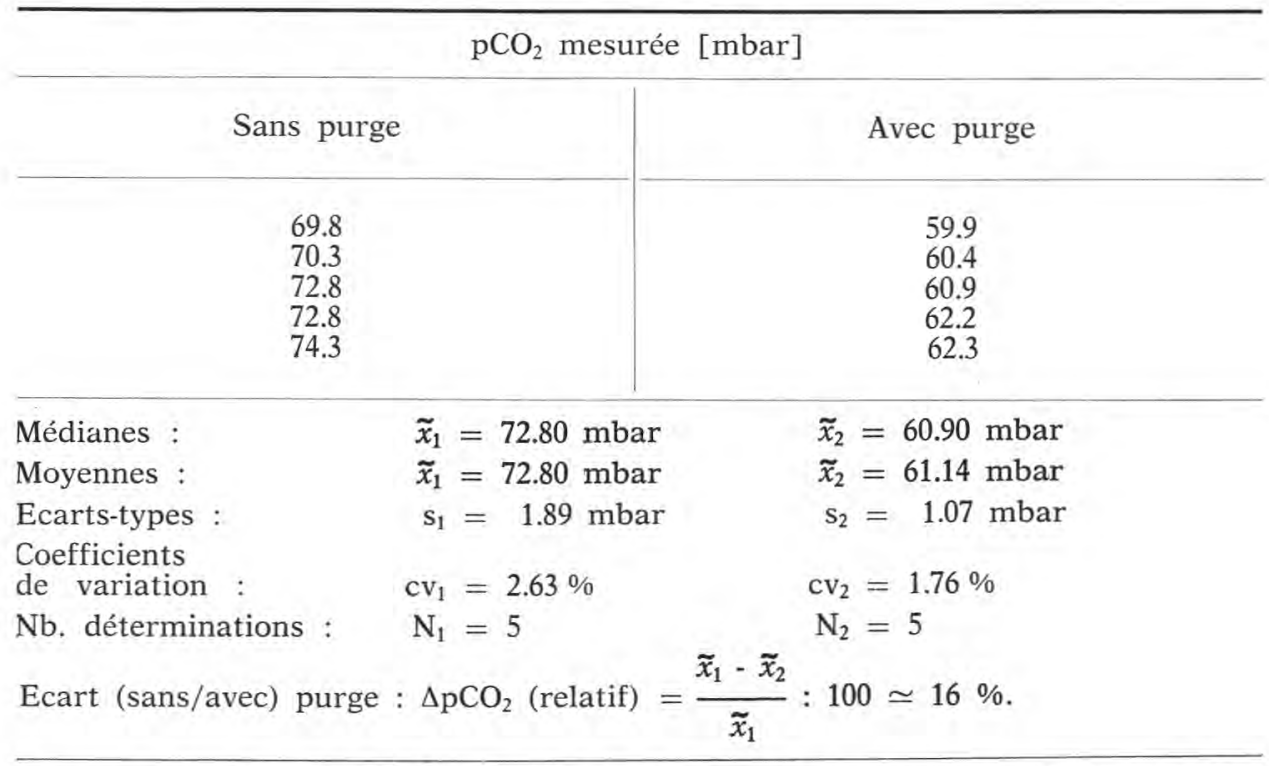

Mesures effectuées sur 10 yoghourts de $180 \mathrm{~g}$, âgés de 1 semaine, provenant d'un lot homogène ; déterminations à simple.

de façon partielle étant donné la viscosité du milieu, par une nouvelle portion de $\mathrm{CO}_{2}$ qui est libérée, cette fois-ci, par l'échantillon lui-même lorsqu'on le secoue pour en briser le gel et en homogénéiser le contenu. Le mode opératoire dit «avec purge » reste néanmoins parfaitement utilisable pour des déterminations relatives à condition de le standardiser de façon stricte, ce qui est le cas ici. L'emploi d'un facteur de correction ( $\mathrm{f}=1,15$ à 1,20 environ) permet d'ailleurs de tenir compte des pertes de $\mathrm{CO}_{2}$ consécutives à cette purge à l'azote et de calculer des valeurs absolues.

Une preuve complémentaire de la validité du mode opératoire dit «avec purge » est la «reproductibilité » des mesures calculée sur la base de la détermination de plusieurs échantillons provenant d'un même lot de fabrication et de stockage. Les résultats du tableau 3 indiquent en effet une « reproductibilité » de l'ordre de 1 à $2 \%$ relatifs « avec purge » $(\mathrm{N}=10$ mesures $)$ au lieu même de 2 à $3 \%$ relatifs «sans purge » $(\mathrm{N}=8$ mesures $)$. Dans tous les cas, la précision est largement suffisante pour la pratique.

En ce qui concerne la «répétabilité » des mesures, calculée ici sur la base de plusieurs mesures consécutives du même échantillon, les résultats mettent en 


\section{TABLEAU III}

Répétabilité et reproductibilité des mesures de la $\mathrm{pCO}_{2}$ (effectuées en double sur 20 yoghourts de $180 \mathrm{~g}$, âgés de 1 semaine, provenant d'un lot homogène, sans et avec purge à l'azote de l'espace de tête des gobelets)

Repeatability and reproducibility of $\mathrm{pCO}_{2}$ measurements (duplicate determinations were performed on 20 one week old yoghurts from a homogeneous lot, with and without flushing of the headspace with $\mathrm{N}_{2}$ )

\begin{tabular}{|c|c|c|c|c|c|c|c|c|}
\hline \multirow{3}{*}{$\begin{array}{l}\text { Paramètres } \\
\text { statistiques }\end{array}$} & \multicolumn{4}{|c|}{$\mathrm{pCO}_{2}$ mesurée (a) (espace de tête non purgé) } & \multicolumn{4}{|c|}{$\mathrm{pCO}_{2}$ mesurée (a) (espace de tête purgé avec $\mathrm{N}_{2}$ ) } \\
\hline & $1^{\mathrm{re}}$ mesure & $2^{\mathrm{e}}$ mesure & \multicolumn{2}{|c|}{ différence } & \multirow{2}{*}{$\begin{array}{l}1^{\mathrm{re}} \text { mesure } \\
{[\mathrm{mbar}]}\end{array}$} & \multirow{2}{*}{$2_{[\mathrm{mbar}]}^{2^{\mathrm{e}} \text { mesure }}$} & \multicolumn{2}{|c|}{ différence } \\
\hline & [mbar] & [mbar] & [mbar] & $\%$ & & & [mbar] & $\%$ \\
\hline & $\begin{array}{c}(44.2)(\mathrm{b}) \\
(63.1)(\mathrm{b}) \\
67.4 \\
71.5 \\
71.7 \\
73.0 \\
73.7 \\
74.0 \\
74.2 \\
74.4\end{array}$ & $\begin{array}{c}(44.8)(\mathrm{b}) \\
(64.5)(\mathrm{b}) \\
68.8 \\
70.0 \\
72.1 \\
72.9 \\
72.3 \\
73.0 \\
73.2 \\
73.4\end{array}$ & $\begin{array}{c}(+0.6) \\
(+1.4) \\
+1.4 \\
-1.5 \\
+0.4 \\
-0.1 \\
-1.4 \\
-1.0 \\
-1.0 \\
-1.0\end{array}$ & $\begin{array}{l}(+1.36) \\
(+2.22) \\
+2.08 \\
-2.10 \\
+0.56 \\
-0.14 \\
-1.90 \\
-1.35 \\
-1.35 \\
-1.34\end{array}$ & $\begin{array}{l}61.7 \\
61.8 \\
62.4 \\
62.5 \\
62.8 \\
63.4 \\
63.6 \\
63.8 \\
63.8 \\
64.4\end{array}$ & $\begin{array}{l}61.1 \\
60.4 \\
61.6 \\
64.8 \\
61.5 \\
62.9 \\
62.6 \\
62.9 \\
63.7 \\
64.2\end{array}$ & $\begin{array}{l}-0.6 \\
-1.4 \\
-0.8 \\
+2.3 \\
-1.3 \\
-0.5 \\
-1.0 \\
-0.9 \\
-0.1 \\
-0.2\end{array}$ & $\begin{array}{l}-0.97 \\
-2.27 \\
-1.28 \\
+3.68 \\
-2.07 \\
-0.79 \\
-1.57 \\
-1.41 \\
-0.16 \\
-0.31\end{array}$ \\
\hline $\begin{array}{l}\text { Nb. dét : } \\
\text { Moyennes : } \\
\text { Ecarts-types : } \\
\text { Médianes : }\end{array}$ & $\begin{array}{l}\mathrm{N}_{1}=8 \\
\widetilde{x}_{1}=72.49 \\
\mathrm{~S}_{1}=2.33 \\
\widetilde{x}_{1}=72.35(\mathrm{c})\end{array}$ & $\begin{array}{l}\mathrm{N}_{2}=8 \\
\widetilde{x}_{2}=71.96 \\
\mathrm{~S}_{2}=1.67 \\
\widetilde{x}_{2}=72.20\end{array}$ & $\begin{array}{c}N=10 \\
\Delta \widetilde{x}=-0.53 \\
\Delta \tilde{x}=-0.15\end{array}$ & $\begin{array}{c}\mathrm{N}=10 \\
\Delta \tilde{x}=0.69 \\
\Delta \tilde{x} / \tilde{x}=0.2 \%\end{array}$ & $\begin{array}{l}\mathrm{N}_{1}=10 \\
\widetilde{x}_{1}=63.02 \\
\mathrm{~s}_{1}=0.92 \\
\widetilde{x}_{1}=63.10(\mathrm{c})\end{array}$ & $\begin{array}{l}\mathrm{N}_{2}=10 \\
\widetilde{x}_{2}=62.57 \\
\mathrm{~S}_{2}=1.42 \\
\widetilde{x}_{2}=62.80\end{array}$ & $\begin{array}{l}\mathrm{N}=10 \\
\Delta \tilde{x}=-0.45 \\
\Delta \tilde{x}=-0.30\end{array}$ & $\begin{array}{c}\mathrm{N}=10 \\
\Delta \tilde{x}=-0,72 \\
\Delta \tilde{x} / \tilde{x}=0,5 \%\end{array}$ \\
\hline
\end{tabular}

(a) Valeurs de $\mathrm{pCO}_{2}$ ordonnées par ordre croissant.

(b) Valeurs anormalement basses (couvercle des gobelets non étanche) non considérées pour l'évaluation des résultats. $\tilde{x}_{1}$ (sans purge) $-\tilde{x}_{1}$ (avec purge)

(c) Abaissement de la $\mathrm{pCO}_{2}$ dû à la purge : $\frac{\tilde{x}_{1} \text { (sans purge) }}{\tilde{x}_{1}}$ 
évidence une très légère $(0,2$ à $0,5 \%$ relatifs) mais néanmoins sensible diminution des valeurs de la $\mathrm{pCO}_{2}$ entre la première et la deuxième mesure du même échantillon, très vraisemblablement due aussi à un lent dégazage du $\mathrm{CO}_{2} \mathrm{de}$ l'échantillon brassé. En pratique, il faut donc se limiter à une détermination en double ou au plus en triple d'un même échantillon.

La « répétabilité 》 des mesures dépend également de la géométrie du récipient et de la qualité du brassage de l'échantillon par l'agitateur pendant la détermination : des pots de verre hauts, étroits et surtout à fond bombé (cf. types a et b) présentent une dispersion des résultats nettement plus importante que des gobelets bas, larges et à fond plus plat (cf. fig. 5).

\section{B. Etude de la plage du $\mathrm{pH}$}

Le principe de la détermination proposée ne peut être correctement appliqué que si le milieu mesuré est suffisamment acide pour que la dissociation en ions $\mathrm{HCO}_{3}$ et $\mathrm{CO}_{3}{ }^{2-}$, inactifs pour la mesure, soit négligeable. Le tableau 1 indique que les $\mathrm{pH}$ des produits considérés, classés par acidité décroissante, sont pratiquement toujours inférieurs à $\mathrm{pH} 4,5$. Cette condition nécessaire à l'application est donc toujours satisfaite ( $>98,7$ sous forme de $\mathrm{CO}_{2}$; cf. fig. 1).

\section{Comparaison avec une méthode de référence}

Un certain nombre d'échantillons du tableau 1 ont parallèlement fait l'objet d'une détermination en double de la teneur totale en $\mathrm{CO}_{2}(\mathrm{mmol} / \mathrm{kg})$ par une méthode de dosage photométrique non dispersif dans l'infrarouge (BOSSET et al., $1980 \mathrm{~b}$ ). Seule est modifiée la partie aliquote, soit d'environ $80 \mathrm{~g}$ utilisés pour les produits laitiers considérés au lieu des $40 \mathrm{~g}$ indiqués pour les échantillons de fromage.

Une comparaison graphique et statistique de ces deux méthodes d'analyse indique une bonne concordance, caractérisée par un coefficient de corrélation $r=0.996(\mathrm{~N}=14$ couples de déterminations) (cf. fig. 3).

La droite de régression calculée est la suivante :

où :

$$
\mathrm{pCO}_{2}=40,19\left(\mathrm{CO}_{2}\right)-0,74
$$
posée ;

$\mathrm{pCO}_{2}=$ pression partielle du $\mathrm{CO}_{2}$ (mbar) mesurée selon la méthode pro-

$\left(\mathrm{CO}_{2}\right)=$ teneur totale en $\mathrm{CO}_{2}$ (mmol/ $/ \mathrm{kg}$ échantillon) mesurée selon BossET et al., $1980 \mathrm{~b}$.

La pente calculée, de 40,19 (mbar. $\mathrm{kg} / \mathrm{mmol}$ ), est hautement significative, Elle n'est autre que l'expression du coefficient de solubilité du $\mathrm{CO}_{2}$ dans le yoghourt à une pression et à une température données, soit ici $1 \mathrm{mmol} \mathrm{CO}_{2} / \mathrm{kg}$ sous 40,19 mbar et $22^{\circ} \mathrm{C}$. En admettant que la loi de Henry soit respectée jusqu'à 1 bar, on en déduit une valeur de $24,88 \mathrm{mmol} \mathrm{CO}_{2} / \mathrm{kg}$ sous 1 bar et $22^{\circ} \mathrm{C}$. Le coefficient de solubilité obtenu ne représente que les deux tiers de celui indiqué pour l'eau, soit $37 \mathrm{mmol} / \mathrm{kg}$ environ pour la même température (WEISs, 1974). 


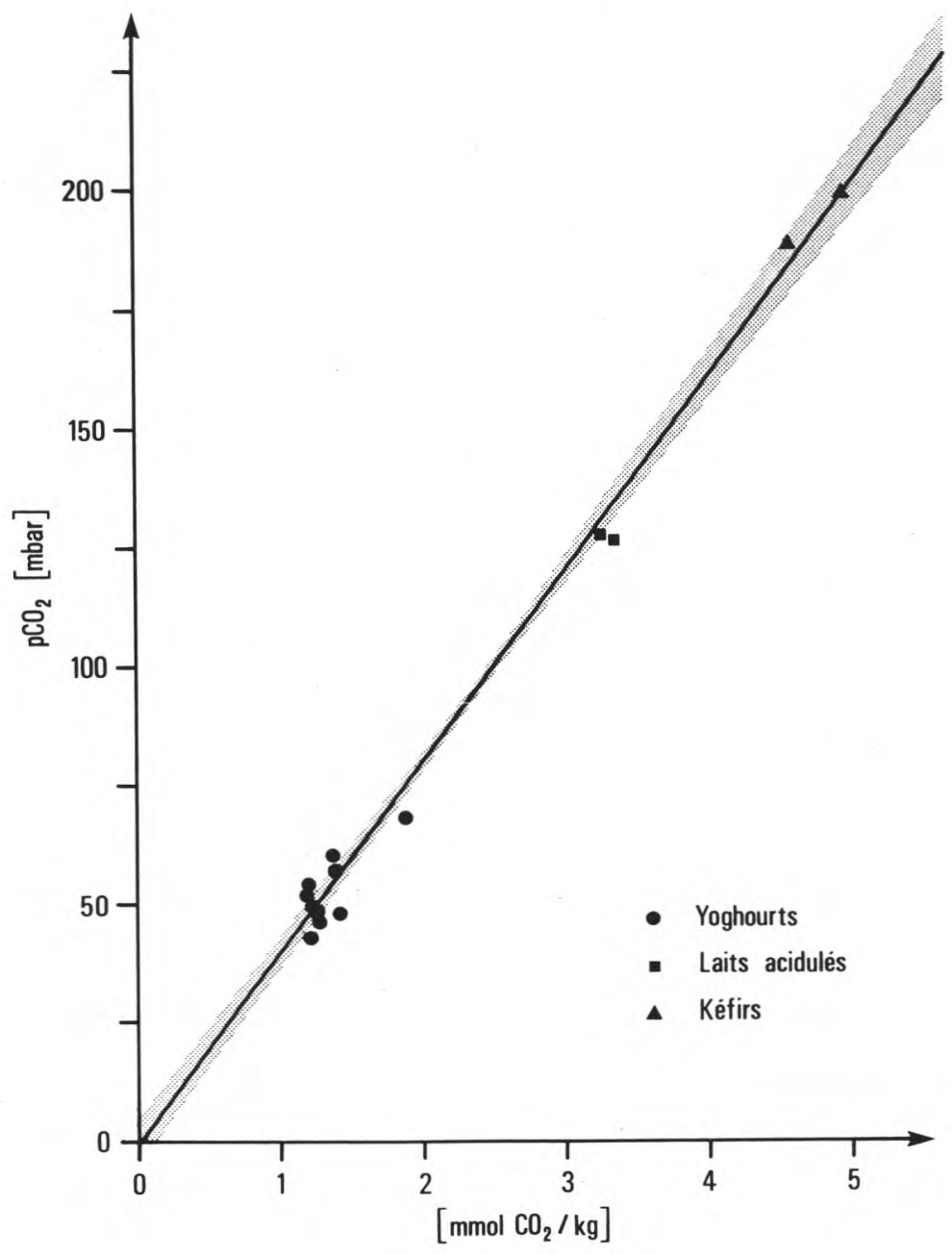

Fig. 3

Comparaison de la méthode proposée utilisant une électrode sensible à la $\mathrm{pCO}_{2}$ avec une méthode photométrique infrarouge non dispersive.

La droite de régression calculée est la suivante : $\mathrm{pCO}_{2}$ (mbar) $\simeq 40,19 . \mathrm{CO}_{2}$ (mmol/kg). La surface ombrée correspond à un intervalle de confiance de $95 \%$ autour de la valeur de $\mathrm{pCO}_{2}$ estimée pour une teneur en $\mathrm{CO}_{2}$ donnée.

Comparison of the proposed methode, using a gas sensing $\mathrm{CO}_{2}$ electrode $\left(\mathrm{pCO}_{2}\right)$, with a non dispersive infrared photometric method $\left(\mathrm{CO}_{2}\right.$ content $)$.

The calculated regression line is : $\mathrm{pCO}_{2}$ (mbar) $\simeq 40.19 . \mathrm{CO}_{2}(\mathrm{mmol} / \mathrm{kg})$. The shaded area corresponds to the $95 \%$ confidence interval around the estimated $\mathrm{pCO}_{2}$ value for a given $\mathrm{CO}_{2}$ content. 
Inversement, l'ordonnée à l'origine calculée, égale à $-0,74$ (mbar), n'est pas statistiquement significative : $\mathrm{F} \alpha \mathrm{\downarrow}=0=0,0746<<4,60$ seuil de confiance de $95 \%$ ). C'est une preuve indirecte de l'absence d'interférence dans le mesurage de la $\mathrm{pCO}_{2}$, le dosage IR non dispersif étant hautement spécifique du $\mathrm{CO}_{2}$. Ces deux méthodes de dosage sont d'ailleurs comparables du point de vue de la répétabilité des mesures (coeff. de variation $=2,7 \%$ pour la méthode de dosage IR, $\mathrm{N}=14$ échantillons en double).

\section{Test de fiabilité}

Afin de déterminer si la réponse et le comportement de l'électrode sont corrects, on a encore mesuré les $\mathrm{pCO}_{2}$ successivement de l'air humide, de l'eau et du yoghourt préalablement saturés par deux gaz de composition connue. Le tableau 4 reproduit les valeurs obtenues. Elles concordent bien avec les valeurs théoriques calculées par la formule suivante :

où :

$$
\mathrm{pCO}_{2}=\left(\mathrm{P}_{\mathrm{B}}-\mathrm{pH}_{2} \mathrm{O}\right) \cdot \frac{\mathrm{C}}{100}
$$

$\mathrm{pCO}_{2}=$ pression partielle du $\mathrm{CO}_{2}$ à la saturation (mbar);

$\mathrm{P}_{\mathrm{B}}=$ pression barométrique (soit $944 \mathrm{mbar}$ );

$\mathrm{pH}_{2} \mathrm{O}=$ pression partielle de vapeur d'eau à $22^{\circ} \mathrm{C}$, soit $27 \mathrm{mbar}$;

$\mathrm{C}=$ concentration $(\% \mathrm{v} / \mathrm{v}) \mathrm{du}$ gaz saturant, soit $\mathrm{CO}_{2}$ dans $\mathrm{N}_{2}$ à $1.00 \% \mathrm{v} / \mathrm{v}$ et à $9.51 \% \mathrm{v} / \mathrm{v}$.

\section{TABLEAU IV}

Test de fiabilité de l'électrode à $\mathrm{pCO}_{2}$ dans des milieux de $\mathrm{pCO}_{2}$ connue

Test of the gas sensing electrode in samples of known $\mathrm{pCO}_{2}$ values

(samples were saturated with gas mixtures of known $\mathrm{CO}_{2}$ content)

\begin{tabular}{c|c|c|c|c}
\hline \multirow{2}{*}{$\begin{array}{c}\mathrm{Gaz} \text { saturant } \\
{\left[\mathrm{V} \mathrm{V} / \mathrm{V} \mathrm{CO}_{2} / \mathrm{N}_{2}\right]}\end{array}$} & \multicolumn{3}{|c}{$\mathrm{pCO}_{2}[\mathrm{mbar}]$ mesurée ou calculée } \\
\cline { 2 - 4 } & Air humide & Eau dist. & Yoghourt & $\begin{array}{c}\text { Val. } \\
\text { théorique }\end{array}$ \\
\hline 1.00 & 8.7 & 9.1 & - & 9.2 \\
9.51 & 83.3 & 83.5 & 86.3 & 87.2 \\
\hline
\end{tabular}

Milieux saturés au moyen de mélanges de gaz de teneur en $\mathrm{CO}_{2}$ connue.

\section{E. Etude des interférences}

Il ressort également du principe adopté pour la détermination de la $\mathrm{pCO}_{2}$ que tout composant gazeux ou volatil acide susceptible de diffuser de l'échan- 
tillon dans la solution intérieure de bicarbonate, à travers la membrane de silicone, peut interférer avec la mesure, Une étude précédente (BOSSET et al., 1980a) a mis en évidence l'importance des acides gras volatils, qui rend par exemple une telle méthode inadéquate pour les dosages dans le fromage, même en technique dite en espace de tête. Dans une récente publication, Lopez (1984) arrive en outre à la conclusion que la réponse de l'électrode est conditionnée plus par l'acidité que par la volatilité des substances interférentes.

Dans les produits laitiers fermentés considérés dans ce travail, les acides lactique et acétique (cf. fig. 1) ont fait l'objet d'une étude particulière vu leur importance quantitative. Les autres acides gras volatils présents, soit pratiquement seuls ceux à courte chaîne et à nombre de carbones pair (Bosset et al., 1986b), sont en quantité insuffisante et/ou à solubilité et à coefficient de diffusion trop faibles dans la membrane pour interférer de façon significative.

Le principal acide contenu dans le yoghourt est l'acide lactique $(<60 \%$ de forme non dissociée, cf. fig. 1), présent à raison de $10 \mathrm{~g} / \mathrm{kg}$ environ (PuHAN et al., 1973), soit environ $20 \mathrm{mmol}$ par pot de $180 \mathrm{~g}$. Trois ajouts de 25,50 et $100 \%$ relatifs ont été effectués en double en ajoutant respectivement $0,5,1,0$ et $2,0 \mathrm{ml}$ d'une solution standard d'acide lactique à $1 \mathrm{~mol} / \mathrm{l}$ (Boehringer Mannheim, réf. 15622) à un aliquot de $18 \mathrm{~g}$ environ de yoghourt préalablement partiellement dégazé (de 5 à 20 mbar de $\mathrm{pCO}_{2}$ ) pour limiter d'une part la perte simultanée de $\mathrm{CO}_{2}$ pendant l'épreuve et accroître d'autre part la sensibilité de cette dernière. Ces ajouts n'ont pas montré la moindre interférence sur la valeur de la $\mathrm{pCO}_{2}$ lue. On peut expliquer à nouveau ce fait par la faible solubilité et par le petit coefficient de diffúsion de ce composé dans la membrane semiperméable en silicone de l'électrode à $\mathrm{pCO}_{2}$.

En ce qui concerne l'acide acétique dont la capacité d'interférer a été démontrée (Bosset et al., 1980a ; Lopez, 1984), sa teneur moyenne a été déterminée préalablement par voie enzymatique (anonyme, 1983) sur 4 échantillons de yoghourt nature du lot considéré. Elle est de l'ordre de $1,7+0,7 \mathrm{mmol} / \mathrm{kg}$ (avec 64 à $92 \%$ de forme active, non dissociée, selon le $\mathrm{pH}$; cf. fig. 1), donc l'équivalent de moins de $25 \mu \mathrm{I}$ d'acide acétique pur par pot de $180 \mathrm{~g}$. Divers ajouts de $25,50,100$ et $200 \mu \mathrm{l}$ de cet acide concentré à des pots de $180 \mathrm{~g}$ de yoghourt nature, soit de respectivement $100,200,400$ et $800 \%$ relatifs, n'ont pas modifié la valeur de la $\mathrm{pCO}_{2}$ lue avant 1'ajout. Il faut recourir à des ajouts nettement plus importants pour observer une interférence. Celle-ci ne se manifeste d'ailleurs que tardivement (après plusieurs minutes) en faisant dériver très lentement le signal mesuré, ce qui semble indiquer qu'il faille commencer par saturer la membrane de silicone (BUCHER, 1985) avant de percevoir une modification au niveau de l'électrode. Aux concentrations naturelles ordinaires en acides gras volatils, notamment en acide acétique des produits laitiers considérés dans ce travail, on peut donc négliger de telles interférences pour la détermination de la $\mathrm{pCO}_{2}$. $\mathrm{La}$ comparaison ci-dessus de la méthode proposée avec un dosage hautement spécifique (par IR non dispersif) confirme d'ailleurs cette indication.

\section{F. Valeurs mesurées pour divers produits fermentés du commerce}

Le tableau 1 indique les valeurs de $\mathrm{pCO}_{2}$ obtenues pour les produits laitiers fermentés considérés prélevés en triple au même moment dans une chaîne de production, mais mesurés à différentes dates pour des raisons pratiques. L'espace de tête des gobelets est préalablement purgé à l'azote. 
On remarque que les $\mathrm{pCO}_{2}$ mesurées se situent approximativement entre 30 et 75 mbar (médiane $=\tilde{x}=50 \mathrm{mbar}$ ) pour les yoghourts âgés de 1 à 2 semaines et nettement au-dessus de 100 mbar pour les produits contenant des levures (type kéfir) capables de produire des quantités importantes de $\mathrm{CO}_{2}$.

On note également que les écarts-types sont sensiblement plus faibles pour les yoghourts dits "brassés» (écart-type moyen $=\widetilde{s}=0.70$ mbar, coeff. de variation moyen $\simeq 1,40 \%, \mathrm{~N}=23$ échantillons) que pour les yogourts dits "fermes » (écart-type moyen $=\tilde{s}=2.33$, coeff. de variation moyen $\simeq 4,6 \%$ $\mathrm{N}=15$ échantillons), ce qui met à nouveau en évidence l'importance du brassage de l'échantillon au moyen de l'agitateur magnétique pendant la mesure ellemềme.

On constate enfin une diminution progressive des valeurs de $\mathrm{pCO}_{2}$ d'une série de mesures à l'autre (chacune en triple) des mêmes sortes d'échantillons. Cette diminution est due à la perte considérable de $\mathrm{CO}_{2}$ à travers l'emballage de polystyrène au cours du temps d'entreposage (cf. fig. 4).

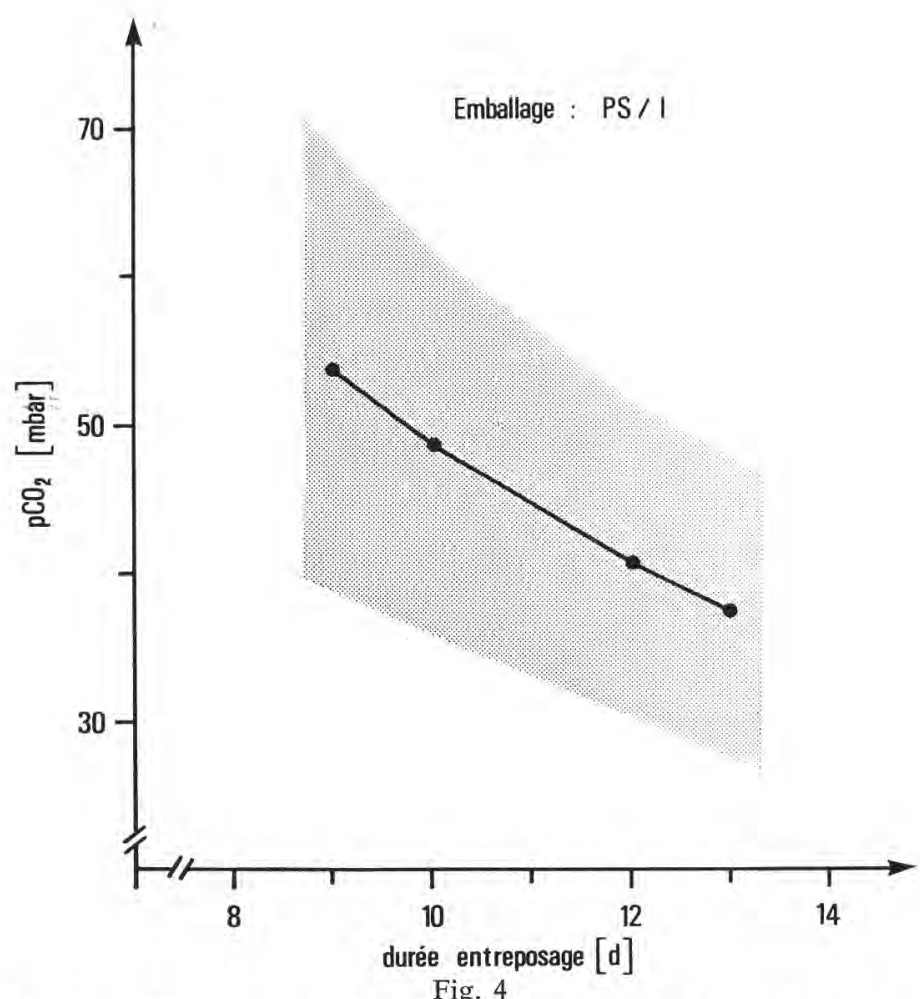

Décroissance de la $\mathrm{pCO}_{2}$ moyenne de yoghourts conservés à $8^{\circ} \mathrm{C}$ due à la perméabilité au $\mathrm{CO}_{2}$ du gobelet en polystyrène.

La surface ombrée correspond à \pm 2 écarts-types (15 échantillons).

Decrease in the mean $\mathrm{pCO}_{2}$ in yoghurts during storage at $8^{\circ} \mathrm{C}$ due to $\mathrm{CO}_{2}$ permeability of polystyrene cup.

The shaded area corresponds to \pm 2 standard deviations (15 samples). 


\section{G. Application à l'étude de la perméabilité des emballages}

La méthode proposée a ensuite été appliquée à la comparaison de la perméabilité des 5 sortes d'emballages (a à e) décrites précédemment (Bosset et al., 1986a). La figure 4 montre l'évolution de la $\mathrm{pCO}_{2}$ d'un même lot de yoghourts au cours de 23 jours d'entreposage consécutifs. Il s'agit des valeurs brutes, non corrigées pour la perte en $\mathrm{CO}_{2}$ de l'espace de tête lors de la purge avec N2 (les valeurs corrigées sont environ $8 \%$ plus élevées pour le verre brun $\mathrm{V} / \mathrm{B}$, respectivement environ 15 à $20 \%$ pour les autres emballages).

En ce qui concerne les trois gobelets en polystyrène (PS/I, PS/B et PS/C), leur perméabilité au $\mathrm{CO}_{2}$ est liée tant à leur structure (solubilité du $\mathrm{CO}_{2}$ ) qu'à leur épaisseur.

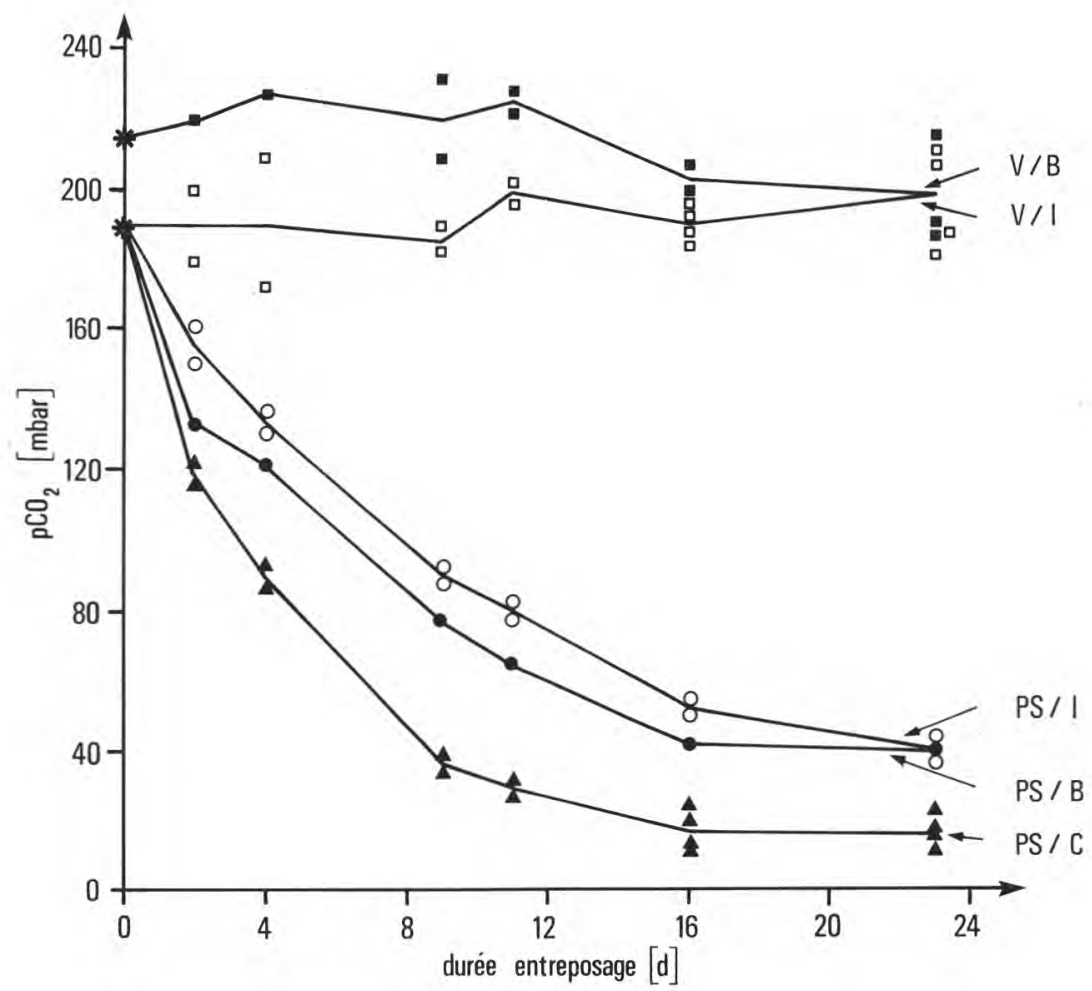

Fig. 5

Evolution de la $\mathrm{pCO}_{2}$ de yoghourts conservés à $8^{\circ} \mathrm{C}$ dans 5 différents emballages pendant 23 jours.

Les valeurs n'ont pas été corrigées pour la perte en $\mathrm{CO}_{2}$ due au rinçage de l'espace de tête des emballages avec $N_{2}$. Les valeurs initiales $\left(^{*}\right)$ ont été extrapolées. Les abréviations utilisées sont les suivantes : $V / B=$ verre brun $; V / I=$ verre incolore $; P S / I=$ polystyrène incolore $; P S / B=$ polystyrène brun ; $P S / C=$ polystyrène cartonné.

$\mathrm{pCO}_{2}$ in yoghurts stored at $8^{\circ} \mathrm{C}$ in 5 different packagings over a period of 23 days.

The values were not corrected for the loss of $\mathrm{CO}_{2}$ due to flushing of the headspace with $N_{2}$. The initial values (*) were obtained by extrapolation. The abbreviations used are : $V / B=$ Brown glass $; V / I=$ Uncoloured glass $; P S / I=$ Uncoloured polystyrene $; P S / B=$ Brown polystyrene ; $P S / C=$ Cardboard polystyrene. 
Quant aux pots de verre (V/B et $\mathrm{V} / \mathrm{I})$, totalement étanches, les valeurs de la $\mathrm{pCO}_{2}$ sont relativement stables et sensiblement parallèles. La différence initiale de la $\mathrm{pCO}_{2}$ dans ces deux types de pots, soit environ $10 \%$, s'explique par leur différence de volume d'espace de tête qui est purgé à l'azote avant la mesure. La très lente diminution de la $\mathrm{pCO}_{2}$ perçue dans le verre brun $\mathrm{V} / \mathrm{B}$ pourrait être due à la faible diffusion du $\mathrm{CO}_{2}$ à travers le couvercle de polyéthylène de ce pot.

Toutes les valeurs initiales sont obtenues par extrapolation. On y observe une bonne convergence des graphes pour les emballages V/I, PS/I, PS/B et PS/C dont les volumes de tête sont sensiblement égaux.

Les concentrations en $\mathrm{CO}_{2}$ dosées par la méthode photométrique non dispersive dans l'infrarouge susmentionnée sur des échantillons identiques (BOSSET et al., 1986a), mais non purgés à l'azote, confirment ces résultats.

\section{Conclusion}

La méthode de mesurage de la $\mathrm{pCO}_{2}$ proposée dans le présent travail répond aux exigences posées dans l'introduction pour être adoptée en routine. Elle est en effet caractérisée par :

- la simplicité de son emploi qui ne nécessite ni réactif dangereux, ni connaissance technique particulière; le $\mathrm{pH}$ naturel des produits laitiers fermentés considérés est en outre suffisamment bas $(\mathrm{pH}<4.5)$ pour ne pas devoir l'ajuster pour l'analyse ;

- le coût relativement modique de l'appareillage nécessaire comparé à celui de la méthode de référence utilisée ;

- une cadence d'analyses de l'ordre de 10 déterminations à l'heure au moins si l'on ne rince pas l'électrode à l'eau entre deux déterminations consécutives; un tel rinçage n'est nécessaire que si l'on détermine successivement des échantillons de nature ou de $\mathrm{pCO}_{2}$ très différentes;

— une répétabilité de l'ordre de 1 à $3 \%$ relatifs au moins si l'on veille à assurer un brassage correct de l'échantillon pendant la mesure et à standardiser le mode opératoire choisi ;

- une bonne spécificité si les produits mesurés ne contiennent pas trop d'acides gras volatils; la comparaison avec la méthode de référence est satisfaisante (coefficient de corrélation $\mathrm{r}=0.996$ pour $\mathrm{N}=14$ paires de déterminations parallèles).

Si l'on effectue simultanément une détermination de la $\mathrm{pO}_{2}$ du même échantillon, il est nécessaire de purger l'espace de tête de ce dernier au moyen d'un flux d'azote. Pour tenir compte des pertes en $\mathrm{CO}_{2}$ qui en résultent, il est nécessaire de recourir à un facteur de correction.

Parmi les applications possibles de cette méthode, on peut citer le contrôle de la fabrication et du stockage $\left(\mathrm{CO}_{2}\right.$ désiré en grande concentration pour le kéfir et les boissons lactées acidifiées analogues; $\mathrm{CO}_{2}$ non désiré en excès dans les yoghourts, car signe de croissance de moisissures ou de levures) et l'étude de la perméabilité de divers emballage à ce gaz.

Reçu le 25 novembre 1985. Accepté pour publication le 10 avril 1986. 


\section{Remerciements}

Les auteurs tiennent à remercier tous ceux qui ont contribué à ce travail, notamment le Dr R. BUCHER de la maison Ingold AG à Urdorf pour l'aimable prêt du matériel analytique utilisé, pour l'intérêt qu'il a porté à cette étude et pour ses précieux commentaires et conseils, ainsi que toutes les entreprises qui ont fourni les échantillons utilisés.

\section{Références bibliographiques}

A.O.A.C. (Assoc. Off. Agric. Chem.), 1980 a. Methods 11.058-11.062. In : Official methods of analysis of the A.O.A.C. 13th ed, A.O.A.C., Washington DC, 190-191.

A.O.A.C..,1980 b. Methods 11.063-11.065. Ididem.

A.O.A.C., 1980 c. Methods 11.066-11.067. Ibidem.

AnoNyme, 1982 a. Manuel d'utilisation de la sonde à $\mathrm{CO}_{2}$. Dr. W. Ingold A.G., CH-8902 Urdorf.

ANONYME, 1982 b. Carbone dioxide electrode. In : Handbook of electrode technology. Orion Research Inc., Cambridge MA 62139, U.S.A., C4-C5.

ANONYME, 1983. Methoden der enzymatischen Lebensmittelanalytik. Arbeitsanleitung zur Analyse von Essigsäure (Acetat), Boehringer Mannheim $\mathrm{GmbH}$, Mannheim.

Blanc B., Bosset J.O., Pauchard J.P., 1980. Etude de la teneur et du dégagement en gaz carbonique du fromage de Gruyère en cours de maturation. Schweiz. Milchw. Forschung, 9, 9-14.

Bosset J.O., Pauchard J.P., Flueckiger E., 1980 a. Le dosage du gaz carbonique dans les produits laitiers : essais de mesure dans le fromage au moyen d'une électrode spécifique. Rapport interne $\mathrm{n}^{\circ} 1$ des sections de technologie et de biophysique. $21 \mathrm{p}$. Station fédérale de recherches laitières, CH-3097 Liebefeld-Berne.

Bosset J.O., Pauchard J.P., Flueckiger E., Blanc B., 1980 b. Nouvelle méthode de dosage du gaz carbonique dans les produits alimentaires et application au fromage. Anal. chim. Acta, 115, 315-321.

Bosset J.O., Daget N., Desarzens C., Dieffenbacher A., Flueckiger E., Lavanchy P., Nick B., PaUchard J.P., TAGLiaferRI E., $1968 \mathrm{a}$. The influence of light transmittance and your permeability of various packing materials on the quality of whole natural yoghurt during storage. In : Food packaging and preservation. M.M. Mathlouth : (ed), Elsevier Appl. Sci. Publ., London, 235-370.

Bosset J.O., Bolomey P., Gmuer W., Gaucex R., 1986 b. Composants volatils de l'arôme du yoghourt nature (en préparation).

BRUNNER H.R., TANNER H., SCHAEPPI R., 1977. Uber eine einfache und exakte Kohlensäurebestimmung in Weinen, Schaumweinen und Süssgetränken. Schweiz. Z. Obst. Weinbau, $113,523-530$.

BUCHER R., 1985. Communication personnelle, c/o Dr W. Ingold AG, CH-8902 Urdorf.

CAPUT A.Yr., 1971. Determination of carbone dioxide in wine. J. Assoc. off. agric. Chem., $54,782-784$.

Charlot G., 1966. $\mathrm{CO}_{2}$ et carbonates. In : Les méthodes de la chimie analytique. Analyse quantitative minérale. $5^{\mathrm{e}}$., Masson, Paris, 667-670.

Flueckiger E., Montagne D.H., StefFen C., 1978. Beitrag zur Kenntnis der $\mathrm{CO}_{2}$-Bildung im Emmentalerkäse vor Beginn der Propionsäuregärung. Schweiz. Milchw. Forschung, 7, 73-78.

KNotT A.C., Belcher C.B., 1975. Determination of carbone dioxide in coal and minerals. Talanta, 22, 751-753. 
Kozlowski E., NAMIESNIK J., 1979. Determination of total carbon and total organic carbon from volatile air pollutants. IV. Comparison of various methods of final determination of $\mathrm{CO}_{2}$. Mikrochim. Acta, 1979, 317-329.

LAdENSON J.A., HuebNeR M., MoRR J.J., 1975. - Continuous real-time monitoring of metabolic parameters in growing bacterial cultures. Anal. Biochem., 63, 59-67.

LIPKA Z., Schopfer J.F., 1972. Méthode simple et rapide de dosage du gaz carbonique dans les vins tranquilles. Rev. suisse vit. arboric., 4, 169-173.

Lonvaud-Funel A., Ribereau-Gayon P., 1976. Le gaz carbonique des vins. I. Dosage à l'aide d'une électrode spécifique. Comparaison avec les méthodes traditionnelles. Connaiss. Vigne Vin, 10, 391-407.

LOPEZ M.E., 1984. Selectivity of the potentiometric carbon dioxide gas-sensing electrode. Anal. Chem., 56, 2360-2366.

Moll M., Flayeux R., Lehuede J.M., 1975. Détermination automatique du $\mathrm{CO}_{2}$ dans la bière. Bios., 6 (3), 85-89.

PAuchaRd J.P., Flueckiger E., Bosset J.O., Blanc B., 1980. $\mathrm{CO}_{2}$-Löslichkeit - Konzentration bei Entstehung der Löcher und - Verteilung im Emmentalerkäse. Schweiz. Milchw. Forschung, 9, 69-73.

Puhan Z., Flueler O., Banhegy M., 1973. Mikrobiologischer Zustand sowie Menge und Konfiguration der Milchsäure der industriell hergestellten Joghurts der Schweiz. Schweiz. Milchw. Forschung, 2, 37-52.

ROBERTSON P.S., 1962. The estimation of carbon dioxide in Cheddar cheese. J. Dairy Res., 321-323.

Shoda M., Ishikawa Y., 1981. Carbon dioxide sensor for fermentation systems. Biotechnol. Bioeng., 23, 461-466.

Simmonds P.G., 1978. Direct determination of ambient carbone dioxide and nitrous oxide with a high-temperature $63 \mathrm{Ni}$ electron-capture detector. J. Chromatogr., 166, 593-598.

Thompson B., 1977. Fundamentals of gas analysis by gas chromatography. Varian Ass. Inc., Palo Alto, California.

VAN DEN BERg M.G., 1979. The partial pressure of carbone dioxide in milk and its relation to the freezing point. Neth. Milk Dairy J., 33, 91-111.

WEISS R.F., 1974. Carbon dioxide in water and sea-water : the solubility of non-ideal gas. Marine Chem., 2, 203-215.

Wiedeking E., Koenig H., KRIEg G., 1980. Einsatz des Prozessphotometers Spectran 657 IR zur $\mathrm{CO}_{2}$-Messung in Faulgasen bei der Klärschlammaufbereitung. ChemieTechnik, 9, 501-503. 